An audit on prescribing practice and risk of serotonin syndrome among patients with chronic pain

Salakan Rai ${ }^{\star}$ and Aizad Yusof

Walsall Healthcare NHS Trust

${ }^{*}$ Corresponding author.

doi: 10.1192/bjo.2021.425

Aims. To determine the incidence of prescribing practice with associated risk of serotonin toxicity among patients with chronic pain conditions.

Background. Serotonin syndrome is a potentially life-threatening condition caused by excessive serotonergic activity, usually from drug interactions. Concurrent use of antidepressants is strongly linked to serotonin syndrome, with recent data revealing record numbers of NHS prescribed antidepressants in 2019. Antidepressant medications are also used in chronic pain management for their anti-neuropathic pain properties. However, it is wellrecognised that a significant number of chronic pain patients suffer from anxiety and depression. This cohort of patients is therefore vulnerable to being exposed to multiple concurrent antidepressant agents, and thus at relatively higher risk of serotonin syndrome compared to other patient groups. Additionally, these patients are likely to be exposed to the concurrent use of antidepressants and certain analgesic agents particularly phenylpiperidine derivatives which increases serotonin toxicity risk.

Method. Medications of patients presenting to a secondary care pain clinic within the last year were looked into. Patients were selected at random by pain management secretaries. Concurrent use of multiple antidepressant agents including Selective Serotonin Reuptake Inhibitors (SSRIs), Serotonin Noradrenaline Reuptake Inhibitors (SNRIs), Tricyclic Antidepressants (TCAs) or Tetracyclic Antidepressant (TeCA) was noted. Additionally, concurrent use of any of these antidepressant agents and phenylpiperidine derivatives such as Fentanyl and Tramadol was noted. Result. Data on medications of 97 patients were collected. A total of 28 patients $(28.8 \%)$ were observed to have at-risk medication combinations. Out of these, five patients were on both SSRI and TCA. Two patients were on both TCA and TeCA. Four other patients were on either a combination of SSRI and SNRI, SNRI and TCA, SSRI and TeCA, or TCA and TCA. Three patients were on both Fentanyl patches and an antidepressant. Fourteen patients were on both an antidepressant and Tramadol. None of these patients were diagnosed with serotonin syndrome; however, it is unclear as to whether these patients experienced milder symptoms of the syndrome.

Conclusion. A considerable number of patients in this group were on medication combinations putting them at risk of serotonin syndrome. Despite no documented patient harm, there is an urgent need for an increased awareness among prescribers on drug interactions which may lead to this syndrome and a subsequent change in prescribing practice.

\section{A survey on psychiatry trainees' experiences of racism}

Aicha Rais ${ }^{\star}$, Richard Burton and Adeel Rauf

Derbyshire Healthcare NHS Foundation Trust

${ }^{*}$ Corresponding author.

doi: 10.1192/bjo.2021.426

Aims. To measure rates of racism experienced and witnessed by trainees training in Derbyshire.

Background. Derbyshire Healthcare Psychiatry trainee workforce comprises 39.1\% white, 52.2\% 'Black, Asian and Minority Ethnic'
(BAME) and $8.7 \%$ undisclosed ethnicity. Racism can affect trainees by increasing risk of poor mental health and psychological distress leading to worse health outcomes. Discrimination, marginalisation and segregation is related to poorer quality education and employment opportunities.

Method. Electronic surveys were sent out via e-mail to trainees in the North and South Derbyshire Hospital sites, accessible online from 11th to 31st December 2020.

Questions asked about personal experiences of racism, witnessing racism from patients and/or staff whilst training in Derbyshire. Trainees were asked if they know how to report incidents and if routinely reported. Trainee ethnicity was recorded.

Result. A total of 56 trainees received a survey request. Response uptake rate $25 \%$ (14 out of 56). Respondents comprised of $36 \%$ white and $64 \%$ BAME doctors. Over one third (36\%) of trainees reported experiencing racism from staff. $64 \%$ of trainees reported experience of racism from patients. There was no report of racism witnessed by staff towards patients. $29 \%$ of trainees reported witnessing both staff on staff racism and patient on staff racism. $93 \%$ of trainees reported witnessing racism from patients to staff. $29 \%$ BAME trainees reported experiencing racism from both staff and patients. 7\% BAME trainees said they experienced racism from staff alone. $36 \%$ of trainees reported experiencing racism from patients only (4 BAME and 1 white trainee). $57 \%$ of trainees do not know how to report racism. 50\% of trainees said they would report racism if they knew how.

Conclusion. Racism remains a barrier affecting the lives of trainees requiring attention. BAME trainees are disproportionately affected by racism, and report witnessing more incidents, from staff and patients in the workplace. There remains an apprehension by doctors to report racism. A departmental presentation has been delivered on racism, unconscious bias, incident reporting process and sources of support. A workshop with the 'Equality Diversity Inclusion' team has been delivered to all trainees with the presence of the Freedom to Speak Up Guardian. Our British Medical Association Local Negotiating Committee Representative has also been informed.

\section{A national CASC course}

Yathooshan Ramesh* and Lisanne Stock

Barnet, Enfield and Haringey NHS Mental Health Trust

${ }^{*}$ Corresponding author.

doi: 10.1192/bjo.2021.427

Aims. There was understandable anxiety from trainees about the transition to the online format of the CASC due to the pandemic. There is also significant variability between trusts in the availability of lectures tailored specifically to the CASC exam. Having recent experience of the CASC exam, including the online format, we developed a free online lecture series. We aimed to address common questions relating to the exam, and selected topics that trainees may find daunting or had less experience with through clinical care. The topics covered were: An Introduction to the CASC, Mental State Examinations, Psychological Therapies, Pharmacology and a Q\&A Session.

Method. The course was designed to tackle areas that trainees often find difficult based on our own experiences as well as surveying course attendees. Prior to the course, we liaised with consultant site tutors \& junior doctor representatives to integrate the course into the local academic programme, and to facilitate promotion of the session to trainees across sites. We subsequently offered registration to trainees nationally. The course was planned and delivered by the organisers through interactive lecture-based 
presentations with handouts, ahead of the January 2021 examinations. Content was based on national guidelines and published research. 5 sessions were delivered with the final session including guest consultant panellists to answer trainee questions. Quantitative and qualitative feedback was collected from the attendees.

Result. 172 doctors registered onto the course, with 44 NHS trusts represented. Doctors from a variety of grades attended, with 55\% CT3s, $17 \%$ Specialty Doctors, $16 \%$ CT2s, $8 \%$ CT1s, $4 \%$ in other roles. $100 \%$ of attendees stated that they would recommend the course to any doctor sitting the CASC. $97 \%$ of attendees rated the course as either 'Excellent' or 'Good'. Qualitative feedback was positive and 3 themes were identified- communication, content and the online format.

Conclusion. The CASC course provided an opportunity to deliver national teaching to trainees based on national guidelines and peer-reviewed research, with a focus on addressing areas that trainees may feel less confident with. The course received significant positive feedback from attendees. The significant number of pre-CT3 trainees attending the course suggests that there may be an interest from this group for further support in developing the complex communication skills that ultimately are assessed by the CASC exam.

An innovative CASC training redesign - 'experience of virtual mock CASC exam'

Jennifer Rankin ${ }^{1 \star}$, Jessica Foster ${ }^{2}$ and Omer Minhas ${ }^{3}$

${ }^{1}$ Aneurin Bevan UHB; ${ }^{2}$ Cardiff \& Vale UHB and ${ }^{3}$ Tonteg Hospital ${ }^{*}$ Corresponding author.

doi: $10.1192 /$ bjo.2021.428

Aims. Cardiff CASC Training (CCT) has provided structured and formal training for the CASC exam for Wales trainees since 2012, in conjunction with Wales Deanery. For the past 8 years CCT has delivered face-to-face mock CASC exams and received excellent feedback from candidates and examiners, in addition to an extremely positive outcome of improvement in CASC pass rate for candidates.

Due to the current COVID-19 pandemic restrictions delivery of the mock CASC examination had to be adapted with the aim of running it remotely via an online platform.

Method. The examinations were run online via Zoom due to its ease of use, including the ability to screen share candidate instructions and assign participants in to breakout rooms. One lead exam coordinator manually rotated candidates around the circuit of 16 stations.

Result. A total of 16 candidates sat the mock exam over two separate sittings. Written feedback was obtained from candidates and examiners. Limitations identified during the initial sitting included high logistical workload for the lead exam co-ordinator and Zoom not being supported by all hospital computer internet browsers, these issues were addressed prior to the second sitting. Feedback from candidates regarding the overall experience of the online exams ranged from 'extremely effective' to 'very effective', this is in line with feedback obtained following previous face-to-face mock exam events CCT has run.

Conclusion. Although online learning may feel very different to the face-to-face interactions we are all used to we are in an era where adaption is necessary. These online mock CASC examinations have been a success and are also in keeping with how the real CASC examination is currently being run by the Royal College of Psychiatrists. CCT are running a further online mock examination to support the next cohort of candidates through their CASC exam in this particularly challenging time.

\section{Peer-led medication education in the time of COVID-19}

Rosa Roberts*

Avon and Wiltshire Partnership Trust; Barnet Enfield and Haringey Mental Health NHS Trust

${ }^{\star}$ Corresponding author.

doi: 10.1192/bjo.2021.429

Aims. To set up an online peer-led medical education programme for core psychiatry trainees during the COVID-19 pandemic.

To determine trainees' views regarding the role of peer-led education in psychiatry.

Method. A peer-led education programme was set up for psychiatry trainees in their third year of core training, held over an online video-conferencing platform. The weekly sessions were organised and led by trainees. Each week a trainee either presented a journal article or a particular psychiatric topic, with a group discussion afterwards.

An online survey was sent to psychiatry trainees that had participated in the programme to determine their views. Close-ended questions were asked as well as open-ended questions for more qualitative responses.

Result. There were 9 peer-led sessions in total, with 11 trainees (out of 18 invited) attending at least one session, and an average of 5 trainees at each session.

Five core trainee psychiatrists responded to the survey following the sessions. All of the respondents found the sessions either "very" or "fairly" useful. $80 \%$ stated that there should be more peer teaching opportunities as part of normal psychiatry training. All respondents found engaging with online teaching either "easy" or "OK".

Open-ended questions showed that respondents found the sessions were useful for: 1) connecting with peers during a difficult time 2) free discussion due to being around peers 3) wide interest and variety of topics. Improvements that could have been made were: 1) more sessions 2) advance knowledge of journal articles being presented.

Conclusion. Peer-led sessions are a useful form of medical education for core psychiatry trainees. During the restrictions brought about by the COVID-19 pandemic they are a way to connect with colleagues during an isolating time. They are easy to organise and access; and can take pressure off medical institutions, whilst having the advantages of allowing trainees to feel they can discuss topics in a more open manner and can follow their own interests.

\section{Immersive psychiatry simulation: a novel course for medical student training}

Kenneth Ruddock* ${ }^{\star}$ Kim Herbert, Catriona Neil, Neera Gajree and Karli Dempsey

NHS Lanarkshire

${ }^{\star}$ Corresponding author.

doi: 10.1192/bjo.2021.430

Aims. Over the last decade, there have been significant developments in the use of simulation for undergraduate medical education. Despite simulation's diverse applications across the medical school curriculum, it has thus far been underutilised within psychiatry teaching. Psychiatric simulation can support students to develop strategies to elicit psychopathology, de-escalate an aggressive patient or perform a risk assessment. Such experiences can be difficult to encounter during clinical placements and may 